\title{
Le Sentiment De Culpabilité Chez Les Infirmiers Face À La Mort De Patients Au CHU Habib Bourguiba Et CHU Hadi Chaker De Sfax (Étude Descriptive Corrélationnelle)
}

\author{
Galaa Telelaz
}

Docteur en psychologie clinique, Psychologue en chef, Université de Tunis El Manar, Faculté de Médecine de Tunis

Feki Ines

Ph.D Professeur agrégé en psychiatrie, Hôpital de Sfax

\section{Masmoudi Jawaher}

Ph.D Professeur en psychiatrie et chef de service, Hôpital de Sfax

Doi:10.19044/esj.2021.v17n15p85

Submitted: 03 March 2021

Accepted: 11 May 2021

Published: 31 May 2021
Copyright 2021 Author(s)

Under Creative Commons BY-NC-ND

4.0 OPEN ACCESS

Cite As:

Galaa T., Feki I. \& Masmoudi J. (2021). Le Sentiment De Culpabilité Chez Les Infirmiers Face À La Mort De Patients Au CHU Habib Bourguiba Et CHU Hadi Chaker De Sfax (Étude Descriptive Corrélationnelle). European Scientific Journal, ESJ, 17(15), 85. https://doi.org/10.19044/esj.2021.v17n15p85

\section{Résumé}

Introduction. Le métier d'infirmier implique une confrontation quasi régulière à la morbidité. Cette confrontation, qu'elle soit occasionnelle ou fréquente, constitue un vécu trop souvent marginalisé est encore trop souvent passée sous silence (Saada et al., 2011). Son indélébilité sur le vécu du professionnel est toutefois une réalité. Elle laisse cependant son empreinte dans le parcours de ces acteurs. En effet, confronté à la mort de son patient, le professionnel de soins infirmiers peut ressentir une certaine culpabilité, du fait d'une nonchalance perçue, ce sentiment incontrôlable et insupportable influe négativement sur sa vie et son travail ce vécu devient handicapant aussi bien sur le plan professionnellement qu'en terme de vie privée. Objectif. Cet article cherche à explorer et évaluer les relations entre le sentiment de culpabilité et les différents facteurs sociodémographiques (genre, âge, niveau d'étude, état civil, ancienneté, nombre de décès) au sein des professionnels de soins infirmiers de l'Hôpital de Sfax, les hypothèses de la recherche stipulent. 


\section{Hypothèse générale :}

- Il existe un lien entre le sentiment de culpabilité chez les infirmiers et leurs facteurs sociodémographiques face à la mort des patients.

Hypothèses spécifiques :

- Il y a un lien entre le sentiment de culpabilité et le sexe chez les infirmiers

- Il existe un lien entre le sentiment de culpabilité chez les infirmiers et leur âge.

- Le niveau d'étude constitue un indicateur de développement du sentiment de culpabilité chez les infirmiers.

- Il existe un lien entre le sentiment de culpabilité des infirmiers participants et leurs nombres de décès des patients sous leurs charges. Méthode. En se basant sur l'inventaire de culpabilité ou Guilt Inventory Scale de Jones et al (2000), cette étude de devis descriptifcorrélationnel fut effectuée sur un échantillon de 131 infirmiers tous genres confondus, dont l'âge moyen est de 32.9 ans. L'avantage de l'outil emprunté, est qu'il offre un score global portant sur le sentiment de culpabilité à mesurer en plus d'apporter une évaluation sur les trois dimensions considérées à savoir l'état, le trait ainsi que les normes morales, ce qui est considérable en termes d'exactitude compte tenu de l'objet étudié. Résultats. L'un des résultats les plus saillants, est que 76\% ( $\mathrm{n}=99$ ) ont exprimé une forte intensité de sentiment de culpabilité. Les résultats ont montré une corrélation positive entre le sentiment de culpabilité et les facteurs suivants : l'âge, le niveau d'étude, l'ancienneté et le nombre de décès. En revanches, une indépendance a été remarquée entre le sentiment de culpabilité, le sexe des participants. Conclusion. En conclusion, les résultats de cette étude témoignent que le sentiment de culpabilité, tel qu'évalué par la Guilt Inventory Scale, est très élevé et en corrélation avec les facteurs ; âge, niveau d'instruction, ancienneté au travail, nombre de décès. Il serait recommandé de prendre en considération ces résultats et venir en aide aux infirmiers par une préparation psychologique à la mort d'un patient afin de ne pas être victime d'une dépression ou un autre trouble.

Mots clés: Sentiment De Culpabilité, Mort D`Un Patient, Infirmier, État De Sentiment De Culpabilité, Trait Du Sentiment De Culpabilité, Normes Morales Du Sentiment De Culpabilité 


\title{
The Feelings of Guilt Among Nurses in Front of the Death of a Patient in the Hospital of Sfax (Descriptive-Correlational Study)
}

\author{
Galaa Telelaz \\ Docteur en psychologie clinique, Psychologue en chef, \\ Université de Tunis El Manar, Faculté de Médecine de Tunis \\ Feki Ines \\ Ph.D Professeur agrégé en psychiatrie, Hôpital de Sfax \\ Masmoudi Jawaher \\ Ph.D Professeur en psychiatrie et chef de service, Hôpital de Sfax
}

\begin{abstract}
Introduction. The nursing profession involves an almost regular encounter with morbidity. This confrontation, whether occasional or frequent, is too often a marginalized experience and is still too often overlooked (Saada et al., 2011). Its indelibility on the professional experience is however a reality. However, it leaves its mark on the career of these actors. Indeed, faced with the death of his patient, the nursing professional may feel certain guilt, due to a perceived nonchalance. This uncontrollable and unbearable feeling has a negative influence on his life and his work. This experience becomes disabling as well on the plan professionally than in terms of privacy. Objective. This article seeks to explore and assess the relationships between the feeling of guilt and the various sociodemographic factors (gender, age, level of education, marital status, seniority, number of deaths) among nursing professionals at the Hospital of Sfax, the research hypotheses state.
\end{abstract}

\section{General hypothesis:}

- There is a causal effect between the feeling of guilt among nurses and their socio-demographic factors in the face of patient death.

Specific hypothesis:

- There is a link between feelings of guilt and sex in nurses

- There is a link between feelings of guilt in nurses and their age.

- The level of education is an indicator of the development of feelings of guilt among nurses.

- There is a link between the feeling of guilt of the participating nurses and their numbers of deaths of the patients under their charge.

Method. Based on the Guilt Inventory Scale of Jones \& al (2000), this descriptive-correlational design study was performed on a sample of 131 nurses of all genders, with an average age of 32.9 years. The advantage of the borrowed tool is that it offers an overall score relating to the feeling of guilt to 
be measured in addition to providing an evaluation on the three dimensions considered, namely the state, the trait as well as the moral standards, which is considerable in terms of accuracy given the object studied. Results. One of the most salient results is that $76 \%(\mathrm{n}=99)$ expressed a strong sense of guilt. The results showed a positive correlation between feelings of guilt and the following factors: age, level of education, seniority and number of deaths. On the other hand, an independence was noticed between the feeling of guilt, the sex of the participants. Conclusion. In conclusion, the results of this study show that the feeling of guilt, as assessed by the Guilt Inventory Scale, is very high and correlates with the factors of age, level of education, length of service at work, number of deaths. It would be advisable to take these results into consideration and help nurses with psychological preparation for the death of a patient so as not to be a victim of depression or another disorder.

Keywords: Feelings of Guilt, Death of a Patient, Nurse, State of Feeling of Guilt, Trait of Feeling of Guilt, Moral Norms of Feeling of Guilt

\section{Introduction}

Le travail d'infirmier confronte de façon immuable à la morbidité, au macabre, à la mort en somme et ce de façon inhérente au métier. Se confronter à la réalité de fin de vie est, pour beaucoup, une épreuve difficile, un véritable dilemme à affronter qui suppose un certain détachement, une force intérieure, de caractère, dirons certains il lui faut aussi connaître la démarche réglementaire pour annoncer aux proches la mauvaise nouvelle (Baussant \& Bercovitz., 2011).

Selon LE Goff. (2006), l'approche de la mort est un soin pour lequel on ne reste pas indemne. C'est une relation à l'autre où les émotions sont activées en permanence. Le soignant ne peut être démuni de caractéristiques humaines dont les émotions font partie. Cette fatalité représentée par la mort peut générer, chez le soignant, un sentiment d'impuissance, de colère et de culpabilité.

Du point de vue de Saada et al. (2011) la confrontation des soignants à la mort, qu'elle soit occasionnelle ou fréquente, est encore trop souvent passée sous silence. Elle laisse cependant son empreinte dans le parcours de ces acteurs.

L'objectif de ce travail est de décrire le degré de sentiment de culpabilité chez les infirmiers à l'Hôpital de Sfax, et ce au travers de l'identification et de l'exploration des relations possiblement existantes entre le sentiment de culpabilité et les différents facteurs sociodémographiques cités dans cette recherche (genre, âge, niveau d'étude, état civil, ancienneté, nombre de décès). 


\section{Problématique}

Face à la mort d'un patient, le soignant est à la fois heurté par la réalité de fin de vie impliquant autrui mais aussi l'impliquant lui-même avec tout ce que cela entraine comme dilemmes, doublement touché par des obsessions intérieures. En tant qu'individu d'abord, il est soumis à la crainte de sa propre mort, de celle de ses proches et au souvenir des décès antérieurs qu'il a pu connaître. De plus, en tant que praticien, l'infirmier peut souffrir d'un échec narcissique important (Maubon., 2011, cité par Fontaine et Wendland., 2015).

Lert et Marne. (1993) expliquent dans leur étude sur "Les soignants face à la mort des patients atteints du sida", que ; "la mort est une violence pour les soignants, générant sentiment d'échec, culpabilité et pour y faire face, conduites d'évitement, conflits ». (p. 201)

Maubon et al. (2011) ont également évoqué la douleur parlé de la douleur du deuil chez l'infirmier, car être confronté de manière récurrente à la mort de «son patient », peut faire souffrir le soignant. La première confrontation avec la mort d'un patient, laisse des souvenirs importants et durables, fait naître des émotions multiples. Être confronté régulièrement et fréquemment à la mort est considéré et vécu comme cause de souffrance psychique. C'est ainsi que certains chercheurs (Lachambre et Marquenet, (2008) ; Tripiana et Lei., (1995), cité dans Maubon et al (2001)) ont étudié l'incidence accrue de la mort d'un patient sur le personnel soignant. Pour Maubon et al., (2011), la mort d'un patient pose la question des compétences ainsi que la culpabilité qui peut en découler. Elle constitue une source majeure de souffrance dans le deuil. Elle crée en plus un sentiment «d'impuissance » qui accompagne les professionnels, notamment celui de ne pas avoir d'impact sur le pronostic vital du partient. Les psychologues invoquent le concept de « sentiment de culpabilité » que Cosnier (1994 : page 140) Définit comme «un sentiment d'avoir commis une faute ou simplement d'en avoir l'intention ».

La culpabilité est à l'origine de $70 \%$ des consultations psychologiques. Elle nous fait ressentir l'impuissance, le sentiment d'être inutile et sans valeur, et bien souvent responsable de tout ce qui va mal... En termes d'impact sur notre écologie personnelle, elle affecte aussi bien la santé mentale que physique donnant lieu à des somatisations ; des maux de tête, des troubles d'estomac ou du sommeil. Elle entraîne également l'impossibilité de se détendre, de l'irritabilité, le blâme une autoflagellation ainsi que et des sautes d'humeur (Blondel, 1986).

Indépendamment des traits de personnalité, il n'est pas évident de comprendre les raisons pour lesquelles certaines personnes se sentent coupables de la perte d'une personne/d'un patient. La psychologie l'explique par le fait que le sentiment de culpabilité trouve souvent son origine dans la prime enfance Baker et al., (2012). L'enfant vient au monde totalement démuni, impuissant, dépendant de ses parents. En outre, le sentiment de 
culpabilité est forcément lié aux valeurs de la société dans laquelle un être grandit. En Occident, par exemple, l'éducation est marquée par des valeurs religieuses qui se fondent, d'une part, sur une longue liste d'obligations et d'interdictions et, d'autre part, sur la crainte de la faute et sur le sentiment de culpabilité (Duhaime., 2016).

La culpabilité ou la honte augmentent les symptômes de stress posttraumatique. Inversement, les interventions qui offrent un sentiment de sécurité, de soutien, de dignité et de contrôle diminuent ces symptômes (Société canadienne de psychologie, 2014). Elle C'est un sentiment aussi répandu que pénible à vivre. Saine lorsqu'elle survient en réaction à une faute, elle peut envahir l'existence et faire vivre dans la confusion se elle devient pathologique (Duhaime., 2016).

Selon Coulter et Pinto., (1995) et Pinto et Priest., (1991), il existe une corrélation négative entre culpabilité et attitudes : plus le niveau de culpabilité est élevé et moins les attitudes sont favorables. L'on note que la corrélation s'inverse lorsque le niveau des appels à la culpabilité est élevé, les niveaux de colère ressentie et d'irritation le deviennent également. L'étude de LavoisierMérieux (2002) a prouvé qu'un message culpabilisant influence positivement les attitudes, les croyances et favorise l'adoption des comportements préconisés. Foulon (2000) évoque le degré de responsabilité qui incombe aux professionnels soignants, une culpabilité très importante, et donc une particularité dans la gestion de leur(s) deuil(s) des patients décédés.

Dans un cadre théorique propre à la psychologie clinique, Larivey (2002), explique le sentiment de culpabilité comme une émotion relative au groupe social qui repose sur la conviction d'une responsabilité personnelle dans un événement fâcheux, dans lequel on n'est pourtant pas toujours intervenu directement, ou au contraire dans lequel on n'aurait pas pu intervenir. Un exemple est celui du malaise ressenti durablement par un infirmier suite à la maladie ou au décès d'un patient. Il est habituel d'éprouver passagèrement un sentiment de culpabilité à l'occasion du travail de deuil d'un patient suivi en service hospitalier (De Peyrelongue, 2011). L'infirmier envahi par ce malaise (sentiment de culpabilité), se prive des possibilités de s'en déprendre (avec d'autres patients) comme s'il s'agissait d'une responsabilité directe. Pour les deux psychologues cliniciens Engel et Ferguson (1999), c'est l'altruisme excessif et mal dirigé qui est parfois source de culpabilité. L'infirmier a un besoin inné de venir en aide aux patients, une tendance à être sauveteur. Il se trouve dans la formule suivante : «pas capable - coupable » et elle fonctionne aussi inversement : « coupable -pas capable ». Quand l'infirmier ne se sent pas capable de faire quelque chose, il se sent coupable de son incapacité. Réciproquement, lorsqu'il se sent coupable, cela le rend souvent incapable d'agir. La culpabilité est donc un sentiment archaïque, 
latent, qui s'habille de différentes facettes et qui guide les comportements (De Peyrelongue, 2011). On en distingue trois formes :

1. la culpabilité réactive : il s'agit d'une "réponse à un acte de transgression des normes d'un comportement acceptable » (Rawlings, 1970, cité dans Chédotal, 2009, page 5)

2. la culpabilité anticipée : elle est "ressentie lorsque l'individu imagine ou anticipe une transgression allant à l'encontre de ses principes internes » (Rawlings, 1970, cité dans Chédotal , 2009, page 5)

3. la culpabilité existentielle : elle "résulte d'une prise de conscience de l'existence d'un écart entre son bien-être personnel et le bien-être d'autrui, il n'y a pas de notion de transgression » (Hoffman, 1982 ; Izard,1977, cité dans Chédotal , 2009, page 6). Certaines personnes ressentent plus facilement cette émotion. Selon Baumeister et al (1994) et Tangney, (1990) les femmes seraient plus sensibles à la culpabilité. Chédotal (2011) ajoute que certains individus seront également plus disposés à ressentir de la culpabilité face à des évènements négatifs. Cette tendance à culpabiliser est un trait de personnalité défini par Mosher (1980) comme : "la structure affective et cognitive qui résulte des expériences passées de situations culpabilisantes et qui vont influencer les actions et les perceptions des situations culpabilisantes actuelles » cité dans Berthe et Chédotal (2018, page 297).

Estryn-Behar (1997 : 11) a mentionné que les travaux cliniques, sur la souffrance psychique au travail en milieu hospitalier, se sont basées sur les éléments de «surcharge psycho-émotionnelle » chez les infirmiers.

De plus, ce sujet a fait l'objet de l'intérêt de plusieurs travaux (Ilhan et al, 2008 ; Vermieren, 2010) sur la charge psychique, en s'appuyant sur des références à la psychanalyse, à la psychopathologie du travail, à l'ergonomie et sur l'expérience d'une recherche/action.

Durand (1999) a donné une importance à la sensibilisation des équipes de soins (la formation est un moyen). Ce travail de sensibilisation vise un changement des habitudes et pratiques qui demande de la patience et beaucoup de temps. Il pense que cette sensibilisation peut aider le soignant à ne pas faire immédiatement (après la mort de son patient) une évaluation négative de luimême, de ses capacités et de la situation en général. Le travail, dans ce cas de figure, doit être thérapeutique. C'est un travail de restauration de la parole. Le pronostic du trauma (mort d'un patient) dépend de la prise en charge immédiate de cet événement classé comme traumatique de la répétition du trauma (Daligand, 2001). Apprendre à gérer sa propre frustration de soignant : on est plus dans le «faire-faire » que dans le «faire». L'évaluation doit être réalisée suffisamment tôt dans l'évolution de la maladie. À ce propos, des 
situations cliniques antérieures difficiles, rediscutées avec les acteurs de soins, peuvent être pédagogiques, ainsi que la recherche clinique.

Pour Daligand (2001), le sentiment de culpabilité protège l'infirmier : il faut l'accepter quand on est soignant, mais il faut travailler en tant que tel soignant sur cette culpabilité imaginaire non conforme à la réalité (la honte, honte d'avoir failli...).

Parvenir à une évaluation régulière de la douleur psychologique (frustration culpabilité...) chez les soignants et à des conduites appropriées de soulagement est un exemple illustre la manière de surmonter cette souffrance qui le handicap dans leur travail (Durand, 1999).

Il est intéressant d'étudier le sentiment de culpabilité car il revient aux émotions morales, en relation avec les normes qu'un individu se fixe. Elster (1999) souligne combien les normes sociales ont une influence immensément puissante sur le comportement. Particulièrement le sentiment de honte qui transcende l'intimité, de plus personnel. Comme le notent Tangney \& Ronda (2003), c'est une émotion qui a une influence certaine sur l'image que nous avons de nous-mêmes et sur la manière dont on pense être socialement perçu. C'est donc une émotion clé de notre comportement, notamment en situation de prise de décision, qui constitue pour Elster (1999), le support des normes sociales.

\section{Cadre théorique}

La culpabilité est un trait universel que chaque être humain éprouve à divers degrés. Elle est liée à la notion de faute, d'erreur, de déviance ou d'échec. Quand on commet ce qu'on perçoit comme un écart à ses principes, on n'ose plus se regarder en face. On se sent coupable et on éprouve de la honte. Puis, lorsqu'on est parvenu à bien se culpabiliser, on s'expose à deux dangers : le premier consiste à se dénigrer, à se faire des reproches, à revenir constamment sur sa faute présumée ; le deuxième, plus courant, comme c'est le cas chez la plupart des criminels, consiste à s'arranger avec sa conscience de manière à ne rien éprouver en se justifiant par toutes sortes d'arguments faciles et arbitraires (Duhaime, 2016).

Le sentiment de culpabilité est généré par le sentiment d'impuissance quand le soignant est confronté à la mort, ce qui traduit que la mort du patient devient une source de souffrance car elle renvoie à l'échec et à la culpabilité (Estryn-Behar, 1997, p.13). La culpabilité survient souvent avant et après le décès. Les personnes atteintes d'une maladie terminale peuvent se sentir coupables si elles se perçoivent comme un fardeau pour leur famille. Les personnes aidantes peuvent se sentir contrariées à cause du temps et de l'énergie qu'elles prennent pour s'occuper de la personne malade, par conséquent, éprouver aussi un sentiment de culpabilité (Société canadienne de la SLA, 1995). 
Dans le cadre de cette étude, la théorie cognitive élaborée par Jones et Kugler (1990) et développée par Jones et al. (2000) a été choisie comme modèle théorique.

Historiquement, les chercheurs ont d'abord distingué la honte de la culpabilité selon le contenu ou la structure des événements qui suscitaient de telles émotions. L'idée était que certains types de situations susciteraient la honte (exemple ne pas faire ce qu'il faut faire) alors que d'autres donneraient naissance à la culpabilité (exemple la mort d'un patient) (Ausubel., 1955). En effet, l'expérience phénoménologique du sentiment de culpabilité, et plus particulièrement l'expérience consciente de l'intensité des sentiments culpabilisants a largement été délaissée par les chercheurs en psychologie (Jones et al., 2000).

La culpabilité serait une expérience "privée" impliquant des aspects de sa propre conscience. Gehm et Scherer (1988) affirment d'ailleurs que la honte est habituellement dépendante d'une exposition publique à sa propre vulnérabilité et fragilité, alors que la culpabilité peut être une entité qui reste secrète à l'intérieur de nous, personne ne connaissant nos normes sociales implicites ou notre responsabilité pour un acte immoral.

La culpabilité touche à un comportement spécifique qui est évalué négativement, sans conséquence toxique au niveau self global. Elle n'affecterait donc pas l'identité profonde de l'infirmier (angl. "self-concept"). Dans la culpabilité, il y a un sens de la tension, du remords et du regret au sujet des choses accomplies, et ce sens motive souvent une action réparatrice : se confesser, s'excuser, réparer le dommage causé. C'est la conceptualisation dominante aujourd'hui (qui est également celle de Lewis) de la culpabilité, pour laquelle il existe un support empirique (Mihaescu., 2006 ).

Bien que les infirmiers aient une capacité à éprouver de la culpabilité à différents moments de leur vie, il y a des différences interindividuelles dans le degré auquel ils sont enclins (trait) à vivre la culpabilité à travers un large panel de situations impliquant les échecs ou les transgressions (Jones et al., 2000). Bien que les sentiments de culpabilité soient suscités par un échec ou une violation des standards moraux, la propension à la culpabilité (en tant que disposition affective) est conceptuellement distincte des standards moraux (définis, comme un ensemble de croyances guidant l'évaluation que l'infirmier fait de ses propres comportements). Il n'est pas clair actuellement, de comprendre en quoi le degré auquel un infirmier ayant une disposition à la culpabilité serait directement une fonction du contenu de ses standards individuels pour ses conduites morales (Jones et al., 2000).

Les chercheurs ont proposé différentes façons de classer la culpabilité et de maintenir une distinction entre la culpabilité morale, la culpabilité trait, et la culpabilité état (Jones et al., 2000 par exemple,). Cette recherche se concentre uniquement sur la culpabilité état et se classifie comme la culpabilité 
passée ou la culpabilité prévue. La culpabilité passée est définie comme transgression psychologique immédiate ou comme un état affectif plus durable suscité par de transgressions passées. La culpabilité anticipée, en revanche, est définie comme la contemplation par un individu d'une possible violation de l'avenir ou la volonté de faire l'expérience d'éventuelles violations à l'avenir. Cette distinction est similaire à Rawlings (1970) et sa classification des culpabilités réactive et d'anticipation.

Après la proposition d'un cadre théorique, Jones et al (2000) ont développé un instrument de mesure de l'intensité du sentiment de culpabilité porté les trois dimensions suivantes :

a. intensité du sentiment de culpabilité (traits) : les émotions interpersonnelles (par exemple, la timidité, la conscience de soi, la colère, etc);

b. intensité du sentiment de culpabilité (état) : un sentiment momentané dans des situations précises;

c. les normes morales, et le réseau de soutien social, les adjectifs pertinents par des amis et des membres de la famille ; les émotions morales qui motivent le respect des normes sociales et des normes personnelles et émergent dans la petite enfance suite au développement de la conscience de soi.

Ces types de culpabilité diffèrent dans les antécédents qui mènent à l'expérience de la culpabilité et de leurs relations avec les autres constructions. Le but de cette recherche descriptive corrélationnelle est de décrire le sentiment de culpabilité des infirmiers face à la mort d'un patient au CHU Habib Bourguiba et CHU Hadi Chaker de Sfax, et d'explorer les relations entre le sentiment de culpabilité et les facteurs cités dans cette recherche.

L'hypothèse principale stipule :

- Il existe un lien entre le sentiment de culpabilité chez les infirmiers et leurs facteurs sociodémographiques face à la mort des patients.

Les hypothèses secondaires expriment :

- Il y a un lien entre le sentiment de culpabilité et le sexe chez les infirmiers

- Il existe un lien entre le sentiment de culpabilité chez les infirmiers et leur âge.

- Il existe un lien entre le sentiment de culpabilité des infirmiers participants et leurs nombres de décès des patients sous leurs charges.

- Il existe un lien entre le sentiment de culpabilité des infirmiers participants et leurs niveaux d'étude. 


\section{Méthode}

\section{Devis de recherche}

Cette étude est une étude transversale, présentant un d'un devis descriptif corrélationnel, qui se base sur l'approche quantitative. Elle a pour but de décrire l'intensité du sentiment de culpabilité chez les infirmiers qui travaillent aux services de chirurgie générale, SAMU, réanimation chirurgicale et hématologie dans les au sein des hôpitaux universitaires Habib Bourguiba et Hedi Chaker de Sfax L'objectif additionnel est de déterminer les relations entre ce sentiment et les facteurs de l'étude.

\section{Caractéristiques des participants}

Nous avons constitué un échantillon de131 infirmiers travaillant dans les hôpitaux «Habib Bourguiba » et «Hedi Chaker» de Sfax. Il se composait de 75 (57\%) infirmières, et 56 (soit 43\%) infirmiers, âgées de 27 à 49 ans avec une moyenne d'âge de 32,9 ans et un écart-type de 7,3. Parmi les participants $55,7 \%(n=73)$ étaient mariés, $41,2 \%(n=54)$ célibataires, et $3,1 \% \quad(n=4)$ divorcés. Le niveau d'instruction est jugé élevé, car 75,6\% ( $\mathrm{n}=99)$ avaient un niveau universitaire et $24,4 \%(n=32)$ un niveau secondaire. $80.1 \%(n=105)$ avaient une ancienneté de moins de 10 ans au travail et $4.6 \%(\mathrm{n}=6)$ avaient une ancienneté plus que 10 ans au travail. De plus, 61\% (n=80) participants avaient vécu moins de 50 décès et $39 \%(n=51)$ avaient vécu plus de 50 décès durant leur carrière professionnelle.

Le tableau suivant nous montre plus de détails sur les caractéristiques de la population :

Tableau $\mathbf{n}^{\circ}$ 1. Facteurs sociodémographiques des participants

\begin{tabular}{|c|c|c|c|}
\hline \multicolumn{2}{|c|}{ Facteurs } & Effectifn & Pourcentage \% \\
\hline \multirow{2}{*}{ Sexe } & Femme & 75 & 57,3 \\
\hline & Homme & 56 & 42,7 \\
\hline \multirow{5}{*}{ Age } & 27 ans & 28 & 21,4 \\
\hline & $28-34$ ans & 70 & 53,4 \\
\hline & $35-41$ ans & 10 & 7,6 \\
\hline & $42-48$ ans & 17 & 13 \\
\hline & 49 ans & 6 & 4,6 \\
\hline \multirow{3}{*}{ Etat civil } & Célibataire & 54 & 41,2 \\
\hline & Divorcé & 4 & 3,1 \\
\hline & Marié & 73 & 55,7 \\
\hline \multirow{2}{*}{$\begin{array}{l}\text { Niveau } \\
\text { d'instruction }\end{array}$} & Secondaire & 32 & 24,4 \\
\hline & Universitaire & 99 & 75,6 \\
\hline \multirow{5}{*}{ Ancienneté } & De 1 à 5 ans & 48 & 36,6 \\
\hline & De 6 à 10 ans & 57 & 43,5 \\
\hline & De 11 à 15 ans & 6 & 4,6 \\
\hline & De 16 à 20 ans & 0 & 0 \\
\hline & De 21 à 25 ans & 20 & 15,3 \\
\hline \multirow{2}{*}{$\begin{array}{l}\text { Nombre de } \\
\text { décès }\end{array}$} & $\leq 50$ & 80 & 61,1 \\
\hline & $>50$ & 51 & 38,9 \\
\hline
\end{tabular}




\section{Méthode d'échantillonnage}

Dans cette recherche la méthode d'échantillonnage probabiliste (aléatoire) stratifié a été utilisée et ce en se basant sur la liste de services dans les hôpitaux où des décès de patients ont été enregistrés. Cette méthode est la plus fréquemment utilisée pour effectuer une sélection aléatoire. Les principales étapes consistaient à :

- définir la population des infirmiers qui travaillent dans les services cités ci-dessus ;

- préparer la liste de tous les services de la population étudiée ;

- certifier que la sélection est aléatoire simple par le choix au hasard.

Comme nous le montre le schéma suivant :

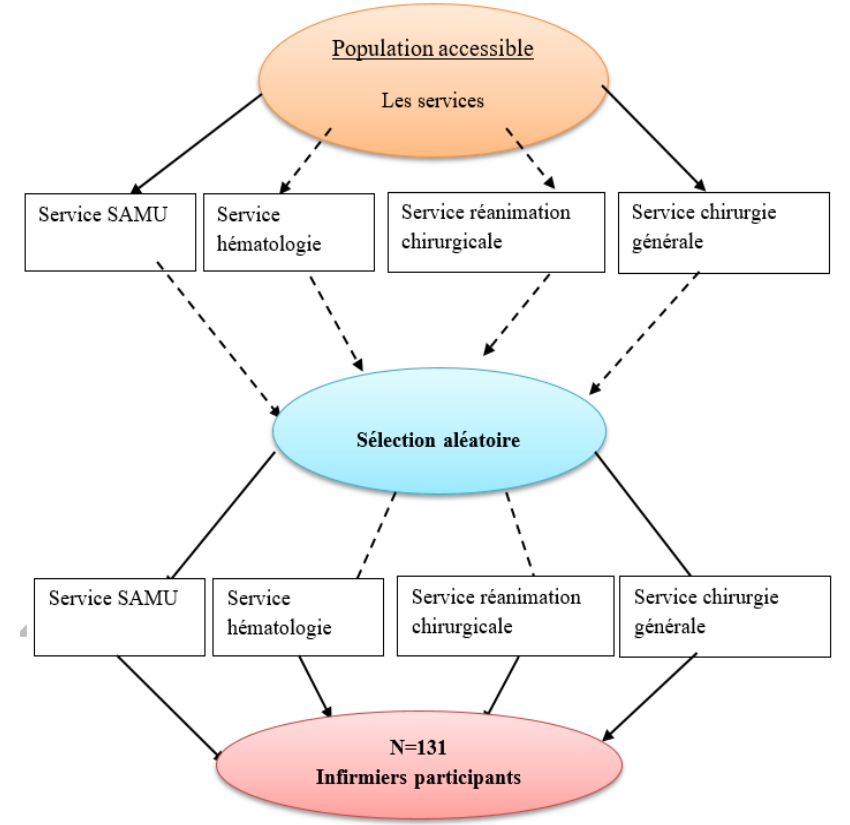

Figure $\mathbf{N}^{\circ} 1$. Méthode d'échantillonnage aléatoire stratifié non proportionnel

\section{L'instrument d'investigation}

Afin de décrire et comprendre l'intensité du sentiment de culpabilité chez les participants face à la mort d'un patient, nous avons choisi la Guilt Inventory Scale (GIS) de Jones et al., (2000) dans sa validation en version française effectuée par Godbout et al., (2005) (Curadeau., 2006). C'est en utilisant une approche cognitive comportementale, que Kugler et Jones (1992) et Jones et al., (2000) ont mis au point une échelle de mesure de l'intensité de sentiment de culpabilité. La Guilt Inventory Scale nous semble l'instrument s'approchant le plus des manifestations émotionnelles et comportementales associées au sentiment de culpabilité décrites. Comme d'autres instruments de 
mesure du sentiment de culpabilité ayant servi de support à des études empiriques, cette échelle a l'avantage de fournir un score total de l'intensité actuelle du sentiment de culpabilité vécu.

Cet instrument cherche à identifier le sentiment de culpabilité chez les infirmiers face à la mort d'un patient en étudiant les trois domaines pertinents (état, trait, normes morales) pour l'évaluation de la culpabilité ; ces dimensions étant la base de création de la conception de l'intensité du sentiment de culpabilité. Il comporte 45 items relatifs à l'évaluation de la culpabilité état, des présents sentiments de culpabilité fondés sur les transgressions actuelles ou récentes restantes (10 items : 4, 16, 17, 19, 30, 33, 36, 37, 43 et 45.). La culpabilité trait, définie comme sentiment continu de culpabilité au-delà des circonstances immédiates, est évaluée grâce à 20 items $(2,5,6,8,9,10,12,14,20,21,23,26,27,29,31,34,35,40,41$ et 44$)$, tandis que les normes morales, qui se définissent soit comme l'abonnement à un code de principes moraux sans référence, soit comme des comportements spécifiques de perception des croyances spécifiques, sont mesurées avec 15 items $(1,3,7,11,13,15,18,22,24,25,28,32,38,39$ et 42). Rappelons toutefois que chacune des dimensions susmentionnées possède une mesure directe qui lui est propre.

L'échelle utilisée dans cet inventaire est une échelle ordinale de type Likert avec une modalité de réponse allant de 1 (fortement en désaccord) à 5 (fortement en accord).

\section{Les scores}

Pour chaque échelle, approximativement la moitié des items est cotée inversement, pour refléter moins de culpabilité ou moins de standards moraux. Les items sont donc côtés de telle sorte que plus le score de chaque item est élevé, plus cela reflète l'ampleur du sentiment de culpabilité ou des standards moraux. Dans le cadre de cette étude, seul le score total cumulé des trois échelles est retenu comme indice de l'intensité de la présence d'un sentiment de culpabilité (Jones \& Kugler.,1993). Il est donc calculé de la façon suivante:

\section{Pour l'état de culpabilité :}

- de 0 à 30 : une faible intensité de sentiment de culpabilité (état);

- de 31 à 50 : une forte intensité de sentiment de culpabilité (état).

\section{Pour le trait de culpabilité :}

- de 0 à 60 : une faible intensité de sentiment de culpabilité (trait);

- de 61 à 100 : une forte intensité de sentiment de culpabilité (trait). 


\section{Pour les normes morales :}

- de 0 à 45 : une faible intensité de sentiment de culpabilité (normes morales)

- de 46 à 75 : une forte intensité de sentiment de culpabilité (normes morales).

\section{Pour les résultats globaux :}

- de 0 à 135 : une faible intensité de sentiment de culpabilité ;

- de 136 à 225 : une forte intensité de sentiment de culpabilité.

\section{Validité et fiabilité de l'échelle de culpabilité (GIS)}

Les fiabilités interne et test-retest de l'instrument ont été démontrées, et la validité a été contrôlée par le calcul de la convergence des moyennes ainsi que la validité discriminante de chaque échelle et ce, en utilisant une série de mesures alternatives à l'intérieur de chacun des trois domaines (c'est-à-dire le trait, l'état, et les standards moraux). La validité a ainsi été établie en démontrant que chaque échelle était plus fortement liée à la moyenne avec des échelles alternatives à l'intérieur de ses domaines qu'avec des échelles alternatives d'autres domaines (Kugler \& Jones., 1992). La Guilt Inventory Scale a aussi été utilisée dans les recherches ciblant la nature interpersonnelle de la culpabilité (Jones \& Kugler, 1993; Jones et al., 1995) et a été reconnue pour son importance en clinique (Dorahy \& Schumaker, 1996; Meehan et al., 1996 cités par Curadeau., 2006).

Jones et al. (1992) rapportent une consistance interne (alpha de Cronbach) pour la propension à la culpabilité de 0,89 , pour l'état de culpabilité de 0,83 , et pour les standards moraux de 0,81 , chez un échantillon de 1041 adultes. La fiabilité test-retest après 10 semaines d'intervalle se situe, pour la culpabilité-trait à 0,72 , pour l'état de culpabilité à 0,56 , et pour les standards moraux à 0,81 (Curadeau., 2006).

\section{Fiabilité de l'échelle de culpabilité dans cette étude :}

Dans cette recherche, nous avons confirmé la consistance interne de l'échelle avec un test de fiabilité qui a montré une cohérence interne très satisfaisante $(\alpha=0.733$; pour $\mathrm{n}=131$, et un nombre d'items $=45)$.

\section{Plan d'analyse}

La saisie et l'analyse statistique des données ont été effectuées sur Statistical Package for The Social Science ${ }^{\circledR}$ (SPSS, version 20.0). Des analyses descriptives, et l'analyse bivariée par le calcul du test de Chi2 et le Coefficient V de Cramer, ont été effectuées tout en considérant les relations significatives lorsque $\mathrm{p}<0,05$. 


\section{Déroulement de l'enquête}

La collecte des données a été réalisée entre Janvier et Avril 2013 à l'hôpital de Sfax. Le questionnaire a été passé en auto administration auprès de 310 infirmiers et infirmières. La durée de réponse au questionnaire était de 20 minutes pour chaque participant.

\section{Considération éthique}

L'autorisation des chefs des services de chirurgie générale, SAMU, réanimation chirurgicale et hématologie à l'hôpital universitaire Habib Bourguiba et Hedi Chaker de Sfax a été demandée avant la collecte des données. Des mesures ont été prises pour respecter les droits et la liberté des participants ; la liberté concernant la participation ou non à l'étude a été clairement énoncée. La confidentialité des données et l'anonymat des participants sont respectés et assurées. Les infirmiers et infirmières ayant donné leur consentement oral à participer à l'étude ont été informés que leur participation est volontaire, anonyme et confidentielle.

\section{Interprétation des résultats}

Dans un premier temps, nous présenterons les analyses descriptives de l'inventaire du sentiment de la culpabilité. Nous présenterons dans un second temps la partie corrélationnelle des variables.

\section{Analyses statistiques descriptive}

Les résultats montrent que les sujets ont une moyenne significativement plus élevée au niveau du trait de culpabilité (61.42 \pm 7.22 ), quel celle trouvé pour la dimension normes morales $(53.65 \pm 7,14)$ et la dimension l'état de culpabilité (31.16 \pm 4.91$)$. Enfin, pour le résultat final, on constate une moyenne égale à $(146,23 \pm 15.89)$.

Les pourcentages obtenus via la mesure de l'intensité du sentiment de culpabilité chez les infirmiers face à la mort d'un patient indiquent que ces derniers démontraient une forte intensité de sentiment de culpabilité, soit 76\%, $\mathrm{n}=99$. 


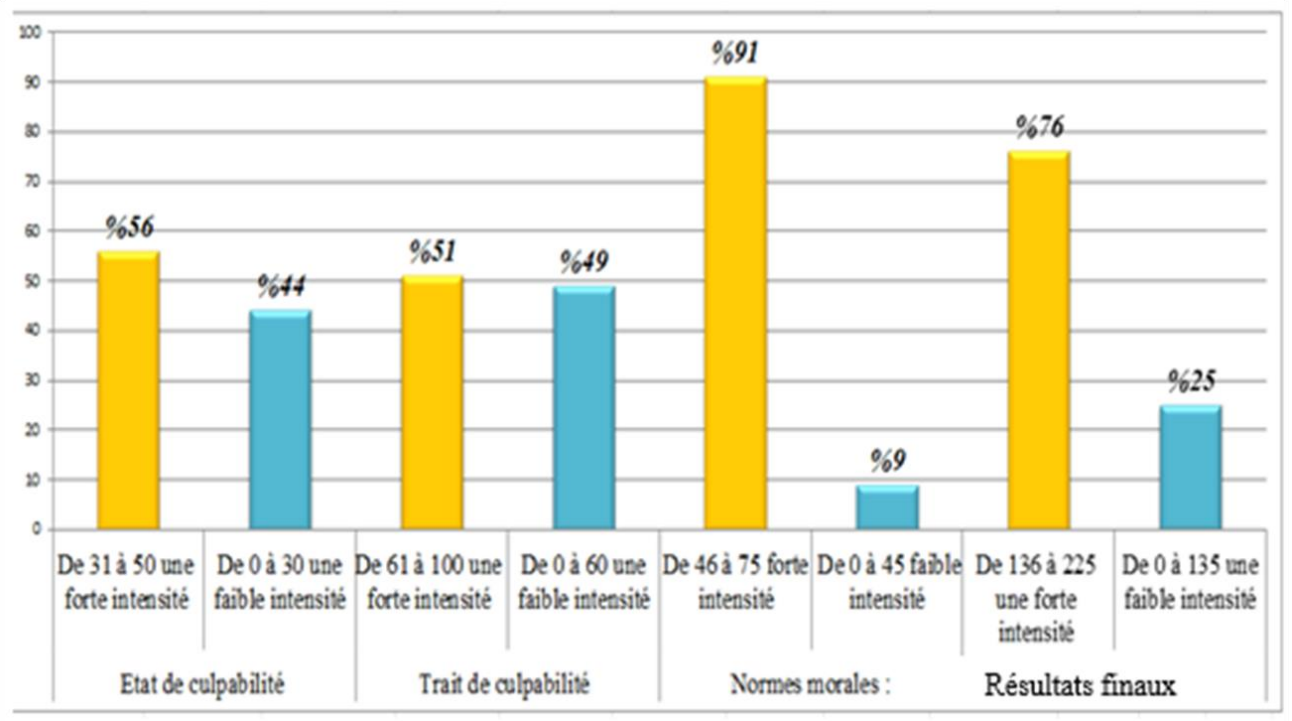

Figure $\mathbf{N}^{\circ} 2$. Distributions des participants selon leur niveau d' 'intensité du sentiment de culpabilité

Ces résultats reflètent le vécu de l'infirmier devant l'effet traumatique de la mort d'un patient dans les services du SAMU, hématologie, réanimation chirurgicale, ou chirurgie générale. Ceci est confirmé par les résultats de l'étude descriptive de Curadeau (2006). Par ailleurs, la moyenne générale obtenue à la Guilt Inventory Scale (GIS) mesurant la présence et l'intensité actuelle d'un sentiment de culpabilité, laquelle est de 123,61 (É.-T. =22,10). De plus, Gil-Monte (2008) a montré que le sentiment de culpabilité a un rôle important dans l'apparition de l'épuisement professionnel des soignants. Il est important de voir les résultats de Gil-Monte (2012) qui révèlent que ce sentiment de culpabilité peut progresser de façon parallèle, allant d'un faible enthousiasme pour le travail à l'insensibilité et l'épuisement psychologique jusqu'à l'indifférence. Cette indifférence est considérée comme un dysfonctionnement. Plutôt qu'efficace, la stratégie d'adaptation essayée peut conduire à un sentiment de culpabilité en raison de l'existence de pensées négatives sur les autres. La manière négative et cynique, à faire face à la situation va les pousser à réessayer de se réadapter avec la perte d'un patient.

La mort d'un patient est un événement traumatisant, et les infirmiers vivent et témoignent la souffrance des patients dues à la maladie. Leurs actes et attitudes ont pour principal objectif d'atténuer le traumatisme suscité par l'annonce ou l'évolution de la maladie. À l'approche de la mort, leur approche holistique et leur fonction accompagnante devient utile et nécessaire.

Les résultats obtenus au niveau de la dimension état de culpabilité décrivent ce sentiment momentané face à la mort d'un patient avec un pourcentage de $56 \%(\mathrm{n}=73)$; les infirmiers répondants vivant un état de 
culpabilité avec une forte intensité. Comme nous le décrit Phaneuf (2014 : page ?), la notion essentielle est que la culpabilité n'est pas un sentiment naturel, mais l'effet de la relation à une instance tierce où le soignant peut, malgré les efforts, montrer une expression faciale momentanée qui peut trahir sa maîtrise des émotions : «on ne peut pas toujours maîtriser une moue, un reniflement.» Dans ce sens, et à propos de l'état des émotions, Berne (1971), fondateur de l'analyse transactionnelle, propose une grille de lecture de nos fonctionnements psychiques appelée « les états du moi ». Ce modèle pratique d'analyse permet de comprendre comment l'infirmier perçoit la réalité de la mort d'un patient, agit et entre en relation avec les autres (infirmiers et patients). Un état du moi est : " un ensemble cohérent de pensées, de comportements, d'émotions, de sensations, de systèmes de défense (identification, projection), de croyances. » (Berne, 1971, p13).

Ces résultats confirment ceux obtenus par plusieurs chercheurs dont Figueiredo-Ferraza et al (2013) qui révèlent l'apparition d'un sentiment de culpabilité état chez les infirmiers et que ce sentiment peut développer chez eux des attitudes négatives en milieu professionnel, en particulier envers les personnes malades avec qui ils établissent des relations de travail.

Il est important de souligner que pour la dimension trait du sentiment de culpabilité, les résultats de l'étude montrent que la moitié des participants, soit $51 \%(\mathrm{n}=67)$, souffre du sentiment de culpabilité (trait) ; ce qui signifie que ce sentiment devient une caractéristique de la personnalité de l'infirmier, vu le nombre des patients décédés (Ekstedt \& Fagerberg,2005; Farber \& Miller, 1981 ; Freudenberger, 1974 ; Maslach, 1982). Il semble que la culpabilité trait des infirmiers est impliquée dans le syndrome de l'épuisement professionnel. D'ailleurs, l'une des causes fréquentes des sentiments de culpabilité chez les professionnels est l'existence négative de réflexions sur les autres et la façon négative et cynique qu'ils ont de traiter les patients (Maslach, 1982). Certains professionnels estiment qu'ils sont devenus très sentimentaux et qu'ils souffrent de ce sentiment de culpabilité qui est devenu un trait de leur personnalité et qui les a conduits à réaffirmer leur engagement à prendre soin de leurs patients (Baumeister et al., 1994 ; Tangney et al., 2007 cités par Figueiredo-Ferraza et al., 2013).

Les résultats exposent un degré élevé de forte intensité de sentiment de culpabilité de normes morales soit $91 \%(\mathrm{n}=119)$. Dans ce contexte, Jones et Kugler (1993) ont expliqués que la culpabilité est conceptualisée comme des sensations désagréables et remords liés à la reconnaissance que l'on a violé ou est capable de violer une norme morale. L'idée défendue par Baumard et Sperber (2007) est que le comportement moral serait plutôt une stratégie adaptée à un environnement social de type contractualiste. En d'autres termes, ces auteurs considèrent qu'une situation morale est une situation d'action collective comme dans le travail infirmier où les intérêts de l'infirmier entrent 
en conflit avec les intérêts collectifs des collègues, et fait le pari que les êtres humains sont naturellement contractualistes, c'est-à-dire qu'ils considèrent spontanément comme "morales" les normes contractualistes (celles que personne ne pourrait raisonnablement rejeter parce qu'elles sont mutuellement profitables). À cela s'ajoute l'idée que dans un environnement où les infirmiers établissent des contrats les uns avec les autres, le comportement moral (qui consiste à suivre les normes contractualistes) s'avère être la meilleure stratégie du point de vue individuel.

Un infirmier qui est considéré comme "bon coopérateur", qui partage équitablement et qui contribue au pot commun sera mobilisé pour mener à bien des actions collectives qui ne posent pas de problèmes et ne développent pas un sentiment de culpabilité, car ces actions apportent des biens qu'on ne peut produire seul, il est donc important d'être rassemblé dans des actions collectives (Baumard, 2007).

\section{Analyse statistique inférentielle}

L'analyse corrélationnelle (khi deux de Pearson) a été réalisée entre les différentes variables de l'étude, afin de mettre à l'épreuve les hypothèses de la recherche qui postulent l'existence de relations significatives entre l'intensité du sentiment de culpabilité et les facteurs sociodémographiques cités dans cette recherche.

Tableau $\mathbf{N}^{\circ} 2$. Les résultats des analyses statistiques corrélationnelles khi2 et V cramer

\begin{tabular}{|c|c|c|c|c|c|}
\hline Facteurs & statistiques & $\begin{array}{l}\text { Normes } \\
\text { morales }\end{array}$ & $\begin{array}{l}\text { État de } \\
\text { culpabilité }\end{array}$ & $\begin{array}{l}\text { Trait de } \\
\text { culpabilité }\end{array}$ & $\begin{array}{l}\text { Intensité de } \\
\text { culpabilité } \\
\text { score final }\end{array}$ \\
\hline \multirow{5}{*}{ Sexe } & Khi-deux de Pearson & 0,477 & 2,905 & 13,395 & 0,477 \\
\hline & $\begin{array}{l}\text { Signification } \\
\text { Valeur de P }\end{array}$ & 0,489 & 0,088 & 0,000 & 0,490 \\
\hline & Valeur de Phi & 0,060 & 0,149 & 0,32 & 0,060 \\
\hline & $\begin{array}{llll}\begin{array}{l}\text { Valeur } \\
\text { cramer }\end{array} & \text { de } & \text { V } & \text { de } \\
\end{array}$ & 0,060 & 0,149 & 0,32 & 0,060 \\
\hline & $\begin{array}{l}\text { Valeur approximative } \\
\text { signification }\end{array}$ & 0,490 & 0,088 & 0,000 & 0,490 \\
\hline \multirow{5}{*}{ Age } & Khi-deux de Pearson & 17,005 & 19,404 & 10,407 & 12,807 \\
\hline & $\begin{array}{l}\text { Signification } \\
\text { Valeur de P }\end{array}$ & $\begin{array}{l}0,002 \\
p<0,05\end{array}$ & $\begin{array}{l}0,001 \\
p<0,05\end{array}$ & $\begin{array}{l}0,034 \\
p<0,05\end{array}$ & $\begin{aligned} 0,012 \\
p<0,05\end{aligned}$ \\
\hline & Valeur de Phi & 0,002 & 0,385 & 0,282 & 0,313 \\
\hline & $\begin{array}{llll}\begin{array}{l}\text { Valeur } \\
\text { cramer }\end{array} & \text { de } & \text { V } & \text { de } \\
\end{array}$ & 0,36 & 0,385 & 0,282 & 0,313 \\
\hline & $\begin{array}{l}\text { Valeur approximative } \\
\text { signification }\end{array}$ & 0,002 & 0,001 & 0,034 & 0,12 \\
\hline \multirow[b]{2}{*}{ État civil } & Khi-deux de Pearson & 6,298 & 5,029 & 12,638 & 3,603 \\
\hline & $\begin{array}{l}\text { Signification valeur } \\
\text { de P }\end{array}$ & $\begin{array}{l}0,04 \\
p<0,05\end{array}$ & 0,081 & $p<0,05$ & 0,165 \\
\hline
\end{tabular}




\begin{tabular}{|c|c|c|c|c|c|}
\hline & Valeur de Phi & 0,219 & 0,196 & 0,311 & 0,166 \\
\hline & $\begin{array}{l}\text { Valeur de } \mathrm{V} \text { de } \\
\text { cramer }\end{array}$ & 0,219 & 0,196 & 0,311 & 0,166 \\
\hline & $\begin{array}{l}\text { Valeur approximative } \\
\text { signification }\end{array}$ & 0,043 & 0,081 & 0,002 & 0,165 \\
\hline \multirow{5}{*}{$\begin{array}{l}\text { Niveau } \\
\text { d'-instruction }\end{array}$} & Khi-deux de Pearson & 4,270 & 1,682 & 3,553 & 7,579 \\
\hline & $\begin{array}{l}\text { Signification valeur } \\
\text { de P }\end{array}$ & $\begin{array}{l}\mathbf{0 , 0 3} \\
p<0,05\end{array}$ & 0,195 & $\begin{array}{l}0,05 \\
p=0,05\end{array}$ & $p<0,05$ \\
\hline & Valeur de Phi & $-0,181$ & $-0,113$ & $-0,165$ & $-0,241$ \\
\hline & $\begin{array}{llll}\begin{array}{l}\text { Valeur } \\
\text { cramer }\end{array} & \text { de } & \text { V } & \text { de } \\
\end{array}$ & 0,181 & 0,113 & 0,165 & 0,241 \\
\hline & $\begin{array}{l}\text { Valeur approximative } \\
\text { signification }\end{array}$ & 0,039 & 0,195 & 0,059 & 0,006 \\
\hline \multirow{5}{*}{ Ancienneté } & Khi-deux de Pearson & 57,75 & 54,492 & 52,121 & 50,351 \\
\hline & $\begin{array}{l}\text { Signification valeur } \\
\text { de } \mathrm{P}\end{array}$ & $p<0,05$ & $p<0,05$ & $p<0,05$ & $p<0,05$ \\
\hline & Valeur de Phi & 0,664 & 0,645 & 0,631 & 0,62 \\
\hline & $\begin{array}{llll}\begin{array}{l}\text { Valeur } \\
\text { cramer }\end{array} & \text { de } & \text { V } & \text { de } \\
\end{array}$ & 0,664 & 0,645 & 0,631 & 0,62 \\
\hline & $\begin{array}{l}\text { Valeur approximative } \\
\text { signification }\end{array}$ & 0,000 & 0,000 & 0,000 & 0,000 \\
\hline \multirow{4}{*}{$\begin{array}{l}\text { Nombre de } \\
\text { décès }\end{array}$} & Khi-deux de Pearson & $112,974 \mathrm{a}$ & $65,308 \mathrm{a}$ & $109,732 \mathrm{a}$ & $91,998 \mathrm{a}$ \\
\hline & $\begin{array}{l}\text { Signification valeur } \\
\text { de P }\end{array}$ & $p<0,05$ & $p<0,05$ & $p<0,05$ & $p<0,05$ \\
\hline & Valeur de Phi & 0,929 & 0,706 & 0,915 & 0,838 \\
\hline & $\begin{array}{llll}\begin{array}{l}\text { Valeur } \\
\text { cramer }\end{array} & \text { de } & \text { V } & \text { de } \\
\end{array}$ & 0,000 & 0,000 & 0,000 & 0,000 \\
\hline
\end{tabular}

\section{Relation entre le sentiment de culpabilité et le sexe}

Concernant le score de l'inventaire de sentiment de culpabilité, une indépendance a été observée entre l'intensité du sentiment de culpabilité et le sexe (khi2 $=0,477, \mathrm{ddl}=1, \mathrm{p}=0,490$ ). Il en est de même pour les deux autres dimensions que sont les normes morales ( $\mathrm{khi} 2=0.477$, $\mathrm{ddl}=1, \mathrm{p}=0.489$ ), et l'état de culpabilité $(\mathrm{Khi}=2.905, \mathrm{ddl}=1, \mathrm{p}=0,088)$. En revanche les résultats de la dimension trait de culpabilité ont révélé une corrélation significative entre cette dimension et le sexe $(\mathrm{Khi}=13.395, \mathrm{ddl}=1, \mathrm{p}<0,05)$. Afin de mesurer l'intensité de cette relation, nous avons calculé le coefficient de $\mathrm{V}$ de Cramer qui a indiqué une faible liaison de sa valeur $(\mathrm{V}=0.320, \mathrm{p}<0,05)$. Ces résultats signifient que malgré le fait que les deux sexes présentent une une intensité de sentiment de culpabilité importante, les femmes semblent plus sensibles aux décès des patients. Ces résultats vont dans le même sens que ceux de l'étude d'Etxebarria et al (2009) qui indiquent que la majorité des études sur la culpabilité concluent que les femmes présentent des scores plus élevés que les hommes. Certaines études indiquent que ce résultat pourrait être influencé par l'exposition fréquente à des événements de nature 
interpersonnelle (Etxebarria et al, 2009). L'instrument utilisé pour mesurer la culpabilité et les modèles de rôle, de sexe et de socialisation peuvent également influencer ces résultats (Benetti-McQuoid \& Bursik, 2005). De cette façon, les hommes développent des rôles féminins (par exemple, en prenant soin).

\section{Relation entre le sentiment de culpabilité et l'âge}

Les résultats de l'étude révèlent une corrélation significative entre le sentiment de culpabilité et le facteur âge pour le score final du test du sentiment de culpabilité (khi2 $=12.807, \mathrm{ddl}=4, \mathrm{~V}=0.313, \mathrm{p}=0.01$ ). Cette corrélation positive est également retrouvée pour les sous-dimensions : normes morales (Khi2 $=17.005, \mathrm{ddl}=4, \mathrm{~V}=0.360, \mathrm{p}=0.002)$, état de culpabilité $(\mathrm{Khi} 2=19.404, \mathrm{ddl}=4, \mathrm{~V}=0.385, \mathrm{p}=0.001)$, et trait de culpabilité (Khi2 $=10.407, \mathrm{ddl}=4, \mathrm{~V}=0.282, \mathrm{p}=0.03)$. Ces résultats correspondent à ceux de Hoffman (1982, 1998, 2000) et Freud (1923/1961, 1930/1961), qui ont trouvé une différence significative entre l'intensité de la culpabilité habituelle et l'âge. Ils ont également suggéré que cette différence peut être liée à une forte anxiété agressive qui augmente progressivement avec l'âge en cas de non consultation ou de suivi psychothérapeutique. De plus, l'étude d'Etxebarria et al. (2009) a montré une différence légèrement significative de l'âge sur le sentiment de culpabilité $(\mathrm{F}(2,272)=2,60, \mathrm{p}=0,076, \eta 2=0,019$, puissance observée $\mathrm{V}=.516$ ). En outre, dans l'étude de Barón et al., Etxebarria, (2018), une corrélation négative a été trouvée entre l'âge et la culpabilité $(\mathrm{R}=-.23, \mathrm{p}<.05)$.

\section{Relation entre le sentiment de culpabilité et le niveau d'instruction}

Le sentiment de culpabilité a été associé au facteur niveau d'instruction par son score final où les résultats statistiques révèlent une significativité (khi2 $=7.579, \mathrm{ddl}=1, \mathrm{~V}=0.241, \mathrm{p}=0.005)$. On peut donc noter que plus leur niveau d'étude est élevé (universitaire), plus les participants présentent un sentiment de culpabilité face à la mort de leurs patients. Cela est étayé par les deux dimensions avec les deux dimensions : normes morales $(\mathrm{Khi} 2=4.270$, $\mathrm{ddl}=1, \mathrm{~V}=0.181, \mathrm{p}=0.03$ ) et trait de culpabilité $(\mathrm{Khi} 2=3.553, \mathrm{ddl}=1, \mathrm{~V}=$ $0.165, \mathrm{p}=0,05)$. Cela n'est pas le cas avec la dimension état de culpabilité où les résultats ont révélé une indépendance (Khi2 $=1.682, \mathrm{ddl}=1, \mathrm{p}=0.195)$.

\section{Relation entre le sentiment de culpabilité et l'ancienneté au travail}

Concernant les liens entre le sentiment de culpabilité et l'ancienneté au travail, on peut observer, là encore, une corrélation significativement élevée et positive pour le score final du test du sentiment de culpabilité en lien avec l'ancienneté des participants ( $\mathrm{khi} 2=50.351, \mathrm{ddl}=13, \mathrm{~V}=0.620, \mathrm{p}<0,05)$, ainsi que pour le lien entre l'ancienneté et les trois dimensions : normes 
morales $(\mathrm{Khi} 2=57.750, \mathrm{ddl}=13, \mathrm{~V}=0.664, \mathrm{p}<0,05)$, état de culpabilité $(\mathrm{Khi} 2=54.492, \mathrm{ddl}=13, \mathrm{~V}=0.645, \mathrm{p}<0,05)$, et trait de culpabilité $(\mathrm{Khi} 2=$ $52.121, \mathrm{ddl}=13, \mathrm{~V}=0.631, \mathrm{p}<0,05)$. Cette relation entre le sentiment de culpabilité et l'ancienneté dans la carrière professionnelle a été bien exposée par Phaneuf (2014), qui explique qu'au début de sa carrière, l'infirmier peut avoir une expérience avec des émotions de tristesse et n'arrive pas à les chasser, car la pensée de la mort revient sans cesse l'habiter. La mort d'un patient provoque chez certains infirmiers un sentiment de peur, d'angoisse répandue. De plus, elle ajoute que l'accompagnement de malades en fin de vie nécessite un deuil particulier, car l'infirmier perçoit avec chaque décès d'un patient un échec de sa mission principale. Pour La chambre et Marquenet (2008), au moment de l'accompagnement, l'infirmier est en lien d'attachement inévitable et normal qui unit le soignant au soigné. De ce fait, le décès de ses patients nécessite un travail de deuil. Ce travail devient lourd et diffère selon la nature de relations et l'intensité des liens affectifs. Ainsi, au cours de sa carrière, l'infirmier fait l'expérience de deuils multiples.

\section{Relation entre le sentiment de culpabilité et le nombre de décès des patients}

Les résultats de l'analyse corrélationnelle suggèrent que le facteur relatif au nombre de décès est davantage associé au sentiment de culpabilité. Ce qui est expliqué par les résultats obtenus comme suit $(\mathrm{khi} 2=91.998, \mathrm{ddl}=$ $16, \mathrm{~V}=0.838, \mathrm{p}<0,05)$, nombre de décès et les trois dimensions ; les normes morales $(\mathrm{Khi} 2=112.974, \mathrm{ddl}=16, \mathrm{~V}=0,929, \mathrm{p}<0,05)$, l'état de culpabilité (Khi2 $=65.308, \mathrm{ddl}=16, \mathrm{~V}=0.706, \mathrm{p}<0,05)$, le trait de culpabilité $(\mathrm{Khi} 2=$ 109.732, ddl $=16, \mathrm{~V}=0,915, \mathrm{p}<0,05)$. Cela signifie que l'intensité du sentiment de culpabilité est forte quand le nombre de décès est élevé. D'ailleurs, Phaneuf (2014) explique que si d'une part le premier défunt dans la carrière d'un infirmier peut le marquer durablement, la répétition de nombreux décès, comme il s'en trouve en soins palliatifs, en soins prolongés et même aux urgences, peut aussi à la longue fortement le troubler. En tant que soignant, il/elle est confronté(e) à la tristesse du départ de personnes qu'il/elle a appris à connaître, qu'il /elle a côtoyé(e)s parfois durant des jours et accompagné(e)s du mieux qu'il/ elle le pouvait dans leurs souffrances physiques et morales. La répétition de rupture de liens par la mort d'un patient peut causer aux infirmiers une certaine usure psychologique qui risque de les fragiliser. Cette auteure ajoute qu' ' il est en effet difficile de refermer la porte de la chambre sans en être profondément troublée, ce qui avec le temps devient difficile à porter. »

\section{Conclusion}

Cette étude a permis de décrire l'intensité du sentiment de culpabilité face à la mort d'un patient chez les infirmiers au deux CHU de Sfax et de 
déterminer et explorer les relations entre le sentiment de culpabilité et les différents facteurs sociodémographiques cités dans cette recherche (genre, âge, niveau d'étude, état civil, ancienneté, nombre de décès). Le choix du sujet est déterminé par l'importance de l'état de l'infirmier et sa souffrance devant le décès d'un patient, surtout que Daligand (2001) a considéré la mort d'un patient comme un pronostic de trauma et que le travail dans ces cas doit être thérapeutique et dépend de la prise en charge immédiate de cet événement classé comme traumatique. D'autre part, Thalmann (2008) affirme que la culpabilité emprisonne, renferme sur soi-même et coupe des autres.

Cette recherche a réussi à répondre aux questions de recherche posées en ce qui concerne la description des concepts de l'étude ainsi qu'à la recherche de relation entre l'intensité du sentiment de culpabilité et les facteurs sociodémographiques. À la lumière des résultats obtenus, nous pouvons déduire que l'intensité du sentiment de culpabilité perçu chez les infirmiers face à la mort d'un patient hospitalisé est très élevée. De plus, les résultats de cette étude ont observé des relations statistiquement significatives entre le sentiment de culpabilité et les facteurs âge, niveau d'instruction, ancienneté au travail, nombre de décès ; ce qui a confirmé les hypothèses mises à l'épreuve. Par contre, on n'a pas trouvé de relation entre le sentiment de culpabilité et le sexe, ce qui signifie que les hommes autant que les femmes souffrent de ce sentiment.

En conclusion, les résultats de cette étude devraient être utilisés pour apporter un soutien aux infirmiers dans l'exercice de leur profession, en leur procurant une aide psychologique, notamment dans les services où on enregistre un grand nombre de décès.

\section{Limites de l'étude}

La conduite de cette étude a rencontré des difficultés dont celle relative à la taille modeste de l'échantillon et qui ne permet donc pas une plus grande généralisation des résultats. En effet, étant donné que seuls 4 services ont participé à l'étude, on peut s'interroger sur la représentativité de l'échantillon. D'autres limites de cette recherche sont dues à sa courte durée, qui n'a pas permis de procéder à des observations plus approfondies, sur un grand nombre de facteurs étudiés, et de réaliser des entretiens avec les participants ayant un sentiment élevé de culpabilité, pour comprendre l'influence dudit sentiment sur leur bienêtre.

\section{Déclaration d'intérêt}

Les auteurs déclarent ne pas avoir de conflits d'intérêts en relation avec cet article. 


\section{References:}

1. Ausubel, D. P. (1955). Relationships between shame and guilt in the socializing process. Psychological Review, 62(5), 378-390.

2. Baker, E., Baibazarova, E., Ktistaki, G., Shelton, K. H., \& Van Goozen, S. H. (2012). Development of fear and guilt in young children: Stability over time and relations with psychopathology. Development and psychopathology, 24(03), 833-845.

3. Barón M. J. O, Etxebarria I. B, Urquijo P. M. A, López S. C \& Aitziber P. J. (2018). Moral emotions associated with prosocial and antisocial behavior in school-aged children. Universidad del País Vasco/Euskal Herriko Unibertsitatea.

4. Baumard, N., \& Sperber, D. (2007). La morale. Published in Terrain, 48.

5. Baumard, N. (2007). Une solution collective aux problèmes d'action collective ; une approche évolutionnaire, cognitive et culturelle des normes morales. In C. Clavien and C. El-Baz. Morale et évolution biologique : entre déterminisme et liberté. Paris Eyrolles

6. Baussant, M., \& Bercovitz, A. (2011). Accompagner le deuil : des repères pour les soignants. 06/2011, vol. 10 , n 3. Pp.125-129. Elsevier Masson.

7. Benetti-McQuoid, J., \& Bursik, K. (2005). Individual Differences in Experiences of and Responses to Guilt and Shame: Examining the Lenses of Gender and Gender Role Sex Role, 53 (2005), pp. 133-142.

8. Berne, E. (1971). Transactional Analysis in Psychotherapy: Analyse Transactionnelle et Psychothérapie. Payot.

9. Berthe, B. \& Chédotal, C. (2018). La culpabilité au travail : La parole aux salariés. Relations industrielles / Industrial Relations, 73(2), 295318. https://doi.org/10.7202/1048572ar

10. Blondel, E. (1986). La culpabilité, une maladie occidentale ? In : Autres Temps. Les cahiers du christianisme social. $\mathrm{N}^{\circ} 10,1986$. pp. $53-$ 59.

11. Burn, E. (1971). Analyse Transactionnelle et Psychothérapie. Edition Payot.

12. Chédotal, C. (2009). L'utilisation de la culpabilité en collecte de fonds : une étude exploratoire des réactions des récepteurs. 8`eme Congrés International des Tendances Marketing, France.

13. Clavien, C. \& El-Bez, C. (2007). Morale et évolution biologique; entre déterminisme et liberté. Lausanne : PPUR Presses Polytechniques et Universitaires Romandes, $338 \mathrm{p}$.

14. Cosnier, J. (1994). La psychologie des émotions et des sentiments, Paris, Retz ; en version actualisée : Emotions et sentiments. Conférences Université Saint-Joseph, Beyrouth, 2004. 
15. Coulter, R. H., \& Pinto, M. B. (1995). Guilt appeals in advertising: What are their effects? Journal of Applied Psychology, 80(6), 697-705.

16. Curadeau, S. (2006). Perturbations alimentaires et abus émotionnel infantile chez des étudiantes universitaires : rôle médiateur du sentiment de culpabilité. Thèse de doctorat à l'université du Québec à Trois-Rivières.

17. Daligand, L. (2001). Culpabilité et traumatisme. In Revue Francophone du Stress et du Trauma, Tome 1(2), 99-101.

18. De Peyrelongue, B. (2011). Le rôle de la culpabilité ressentie dans le consentement à payer : application aux achats pour l'enfant et à l'achat de produits alimentaires bio. Thèse de doctorat, de Bourgogne). Repéré à :https://tel.archives-ouvertes.fr/tel-00737878/document

19. Duhaime, B. (2016). La culpabilité. Dans Ombres et Lumière Publié sur : www.larchedegloire.com.

20. Durand, H. (1999). Soins palliatifs et accompagnement. ADSP $n^{\circ} 28$ septembre 1999. Paris.

21. Ekstedt, M., \& Fagerberg, I. (2005). Lived experiences of the time preceding burnout. Journal of Advanced Nursing, 49(1), 59-67

22. Elster, J. (1999). Alchemies of the mind : Rationality and the emotion . Combridge, p. 145ss.

23. Engel, L., \& Ferguson, T. (1999). La culpabilité Broché ne plus la subir ne plus en souffrir. Editeur Le Jour. 293 pages.

24. Estryn-Behar, M. (1997). Stress et souffrance des soignants à l'hôpital : reconnaissance, analyse et prévention. Paris : Éd. ESTEM, 1 vol. (245 p.)

25. Etxebarria, I., Ortiz, MJ., Conejero, S., \& Pascual, A. (2009). Intensity of habitual guilt in men and women: differences in interpersonal sensitivity and the tendency towards anxious-aggressive guilt.in Span J Psychol. 2009 Nov;12(2):540-54.

26. Farber, B. A., \& Miller, J. (1981). Teacher burnout : A psychoeducational perspective. Teacher College Record, 83, 235-243.

27. Figueiredo-Ferraza, H., Gil-Montea P.R., \& Grau-Alberolab E. (2013). Les propriétés psychométriques du Spanish BurnoutInventory (SBI): adaptation et validation auprès d'un échantillon portugais . Valencian International University, Valencia, Spain,in Revue européenne de psychologie appliquée 63 (2013) 33-40.

28. Fontaine, E., \& Wendland, J. (2015). Analyse de la relation soignantbébé et du deuil des soignants en néonatologie. Dans Devenir 2015/1 (Vol. 27), pages 31 à 52.

29. Foulon, M., (2000). Le cadre face aux rites de passages des soignants. Recherche en soins infirmiers, 63, 52-72. 
30. Freudenberger, H. (1974). Staff burnout. Journal of Social Issues. 30 : 159-65.

31. Gehm, T. L., \& Scherer, K. R. (1988). Relating situation evaluation to emotion differentiation: Nonmetric analysis of cross-cultural questionnaire data. In K. R. Scherer (Ed.), Facets of emotion: Recent research (pp. 61-77). Hillsdale, NJ, US: Lawrence Erlbaum Associates, Inc.

32. Gil-Monte, P. R. (2008). Magnitude of relationship between burnout and absenteeism : a preliminary study. Psychological Reports 103 : 465-.8.

33. Gil-Monte, P. R. (2012). Influence of guilt on the relationship between burnout and depression. European Psychologist. 17(3) : 231-6.

34. Hoffman, M.L. (1982), Development of prosocial motivation : empathy and guilt. in N. Eisenberg (coord.), The Development of Prosocial Behavior. London, Academic Press, 281-311.

35. İlhan MN, Durukan E, Taner E, Maral I, Bumin MA. (2008). Burnout and its correlates among nursing staff: questionnaire survey. J Adv Nurs. (2008) Jan;61(1):100-106. doi: 10.1111/j.13652648.2007.04476.x. Epub 2007 Nov 22. PMID: 18034813.

36. Izard, C. E. (1977). Human emotions, New York, Plenum Press.

37. Jones, WH., Schratter, AK., \& Kugler, K. (2000). The Guilt Inventory. In Psychol Rep. 2000 Dec; 87(3 Pt 2):1039-42.

38. La chambre, V., \& Marquenet, C. (2008). Le travail de deuil du soignant en oncologie pédiatrique. CNDR Soin Palliatif - Centre National de Ressources Soin Palliatif

39. Larivey, M. (2002), La puissance des émotions, Comment distinguer les bonnes des mauvaises. Editeur : Pocket, Collection : Pocket. Pages : 376 pages

40. Lavoisier-Mérieux, L. M. (2002). De l'efficacité des stratégies de communication publicitaires menaçantes : une étude de la relation entre émotions négatives et persuasion dans le domaine de la lutte contre l'insécurité routière. Actes du 2ème Congrès International des Tendances du Marketing, Paris

41. Le Goff, J. P. (2006). Evaluation, l'intériorisation des normes. Revue Projet 2006/2 (nº 291), Pp 67 à 72 . Éditeur : C.E.R.A.S

42. Lert, F., Marne, M.J., \& Gueguen, A. (1993). Evolution des conditions de travail des infirmières des hôpitaux publics de 1980 à 1990. Rev Epidemiol Saute Publ 41:16-29.

43. Macaulay, J., \& Berkowitz, L. (1970). Altruism and helping behavior - Social psychological studies of some antecedents and consequences. New York, Academic Press, 163-177. 
44. Maslach, C. (1982). Burnout The Cost of Caring. Englewood Cliffs: Prentice Hall. 192 p.

45. Mihaescu G. (2006). Réflexions sur la culpabilité dans la psychopathologie, Paris, ILV.

46. Phaneuf, M. (2014). Qualité de vie au travail ou qualité des soins faut-il vraiment faire un choix?. http://www.prendresoin.org/wpcontent/uploads/2014/09/Qualite-de-vie-au-travail-ou-qualite-dessoins.pdf

47. Pinto, M. B., \& Priest, S. (1991). Guilt appeals in advertising: An exploratory study. Psychological Reports, 69, 375-385

48. Rawlings, E.I. (1970). Reactive guilt and anticipatory guilt. in altruistic behavior, in J. Macaulay et L. Berkowitz (coord.), Altruism and helping behavior - Social psychological studies of some antecedents and consequences, New York, Academic Press, 163-177.

49. Saada, B., Maubon, M., \& Nectoux L, M . (2011). Mort des patients, professionnels en deuil ?. Responsabilité. 12/2011, vol. 11, n 44, Pp. 29-31.

50. Société canadienne de la SLA. (1995). Faire face au deuil, Stratégies destinées aux personnes aux prises avec la SLA.

51. Société canadienne de psychologie. (2014). Série « La psychologie peut vous aider » : L'état de stress post-traumatique, Ottawa (Ontario) K1P 5J3.

52. Tangney, J. P. \& Ronda, La D. (2003). Shame and Guilt. HARD COVER PAPER BACK. 272 Pages.

53. Thalmann, Y. A. (2008). Au diable la culpabilité. Editeur Jouvence.

54. Vermeiren E (2010). Le burn-out chez les soignants et dans les équipes. Urgences, communication présentée au 4é congés de la société française de Médecine d'Urgence Paris Accès le 20 juin 2011, http://www.sfmu.org/urgences2012/urgences2010/donnees/fsrecherche/fs_009_vermeiren.htm (dernier accès le 10 juin 2012) 